\title{
Numerical Solution of a Parabolic Free Boundary Problem Arising in Statistical Decision Theory
}

\author{
By Gary G. Sackett*
}

\begin{abstract}
A parabolic free boundary problem which arises in a statistical decision setting is reduced to a free boundary problem for the heat equation which is amenable to numerical solution by the method of lines. An algorithm is given and the apparently globally converging results are compared with the asymptotic expansions of $\mathrm{H}$. Chernoff and J. Breakwell.
\end{abstract}

Introduction. In a series of papers [1], [2], [3], Herman Chernoff and John Breakwell describe a statistical decision problem, relate it to a parabolic free boundary problem, and give an asymptotic solution for that free boundary problem.

In this paper, the method of lines (sometimes referred to as the Rothe technique, after a paper of Erich Rothe [5]) is employed to solve numerically a free boundary problem for the heat equation which is equivalent to the free boundary problem of Chernoff and Breakwell. As will be pointed out later, the asymptotic results of Chernoff and Breakwell are valid strictly locally; the method of lines technique employed here appears to converge globally.

Background. For those readers who might be interested in a skeletal view of the background, a brief description of the original statistical decision problem is given. A more detailed account is to be found in [2].

Consider a Wiener-Lévy stochastic process $\chi(\tau)$ and the associated process $\xi(\tau)$ having drift $\mu$,

$$
\xi(\tau)=\chi(\tau)+\mu \tau
$$

where $\mu$ is an unknown constant whose sign is to be determined. $\mu$ is to be considered as a random variable with known a priori normal distribution. Starting at some time $\tau_{0}, \xi(\tau)$ is observed for a period of time and then, according to a prescribed stopping rule, observation ceases and $\mu$ is hypothesized as positive or negative according as the sign of $\xi$ is positive or negative at that instant. Since there is to be assessed a cost of observation and a cost of making an incorrect decision, this prescribed stopping rule should be chosen in such a way as to minimize the expected cost of the entire operation.

Specifically, assign to each point $(\xi, \tau)$ a Bayes risk $B(\xi, \tau)$, defined by

$$
\begin{aligned}
B(\xi, \tau)= & E\{\text { cost of an incorrect decision } \mid \xi(\tau)=\xi\} \\
& +E\{\text { cost of continued testing from }(\xi, \tau)\},
\end{aligned}
$$

Received August 17, 1970.

AMS 1970 subject classifications. Primary 65N99; Secondary 62C10, 35K05, 65M20.

Key words and phrases. Free boundary, method of lines, Bayes risk.

* Parts of this paper appeared in the author's doctoral dissertation at the University of Southern California; the bulk of the results were supported by NSF Grants GU-2582 and GP-12365.

Copyright @ 1971, American Mathematical Society 
where $E$ is the expected value operator. Then the problem becomes one of dividing the upper half-plane $\tau \geqq \tau_{0}$ into two regions, a continuation region and a stopping region, in such a way as to uniformly minimize $B(\xi, \tau)$. These regions may be viewed as being defined by two boundaries $\xi=\sigma_{i}(\tau), i=1,2$, as indicated in Fig. 1 .

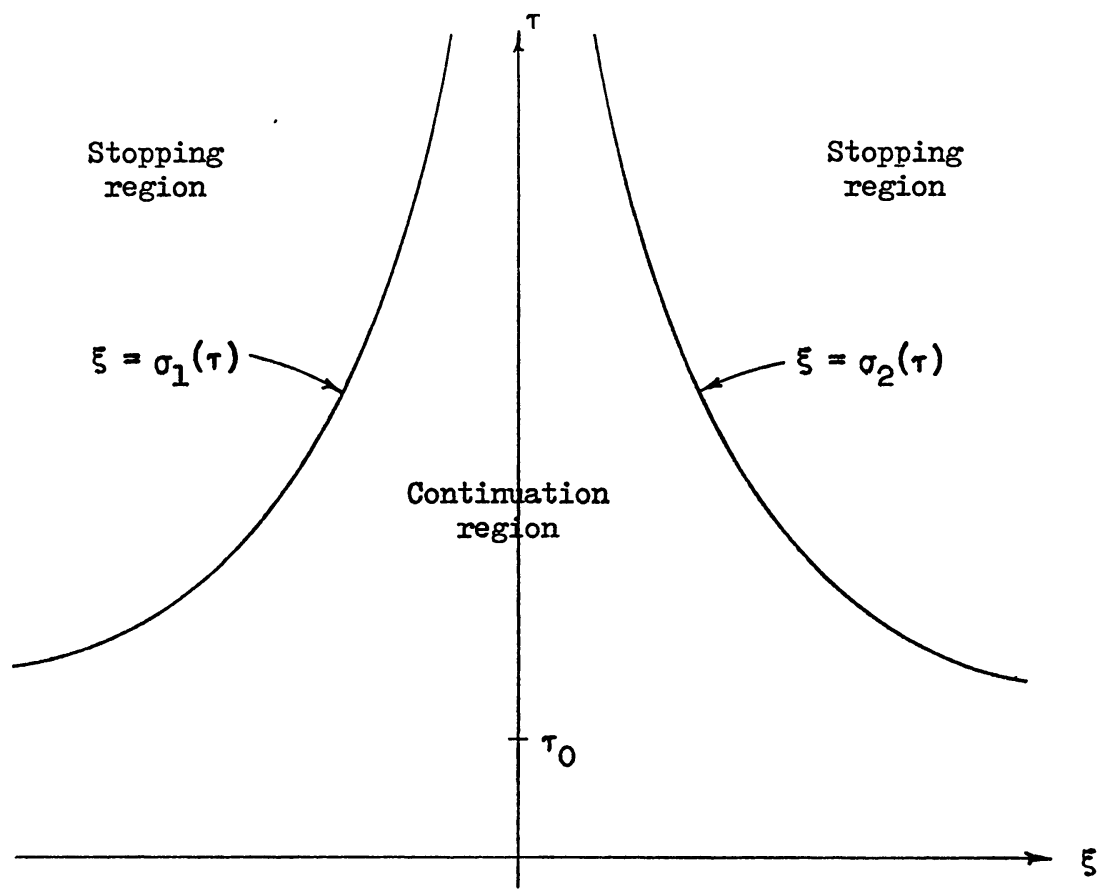

FIGURE 1

In particular, let the cost of an incorrect decision be proportional to $|\mu|$, and assume that the cost of observation is constant per unit time. Then, Chernoff has shown in [2] that, after suitable normalization, $B$ must satisfy the parabolic equation

$$
\frac{1}{2} B_{\xi \xi}+\frac{\xi}{\tau} B_{\xi}+B_{\tau}+1=0
$$

in the continuation region. The symmetry of the problem leads to the observation that $\sigma_{1}(\tau)=-\sigma_{2}(\tau)$ and further, the definition of $\sigma$ as the stopping boundary gives rise to the boundary condition

$$
B(\sigma(\tau), \tau)=D(\sigma(\tau), \tau)
$$

where $D$ is the known function given by

$$
D(\xi, \tau)=\tau^{-1 / 2} \psi\left(\xi \tau^{-1 / 2}\right)
$$

where

$$
\begin{aligned}
\psi(\alpha) & =\varphi(\alpha)+\alpha \Phi(\alpha)-\alpha, & & \alpha>0, \\
& =\varphi(\alpha)+\alpha \Phi(\alpha), & & \alpha \leqq 0,
\end{aligned}
$$

and $\varphi, \Phi$ are density and distribution functions, respectively, for a normal $(0,1)$ distribution. 
Chernoff has given an argument, related to and reported by Sherman [7], that if such a minimizing curve exists, the so-called "smooth-pasting" condition

$$
B_{\xi}(\sigma(\tau), \tau)=D_{\xi}(\sigma(\tau), \tau)
$$

must also be satisfied. The problem is rounded out with the boundary condition

$$
B_{\xi}(0, \tau)=0,
$$

deduced from symmetry considerations.

Metamorphosis. The problem (1), (2), (3), (4) is seen to be a parabolic free boundary problem for the determination of $B$ and $\sigma$. We shall metamorphose this problem into a problem which is susceptible of solution by the method of lines by first enlisting the transformation

$$
x=\xi / \tau, \quad t=1 / 2 \tau
$$

as suggested by Sherman in his report [7] dedicated to the analytic examination of our problem.

Then, putting

$$
u(x, t)=B(\xi, \tau)-D(\xi, \tau)+\tau, \quad s(t)=2 \tau \sigma(1 / 2 \tau),
$$

a free boundary problem for the heat equation emerges. Namely,

$$
\begin{aligned}
u_{x x} & =u_{t}, & & 0<x<s(t), \\
u_{x}(0, t) & =\frac{1}{2}, & & t>0, \\
u_{x}(s(t), t) & =0, & & t>0, \\
u(s(t), t) & =1 / 2 t, & & t>0 .
\end{aligned}
$$

This problem, save one critical distinction, is of the type considered in [6]. That distinction is found in condition (7d), which makes the problem singular at $t=0$ $(\tau=\infty)$.

Since such a singularity is somewhat distasteful, an attempt to remove it is made by considering the following decomposition of $u$ :

$$
u(x, t)=w(x, t)+v(x, t)
$$

where $w$ is to have the form

$$
w(x, t)=t^{-1} \theta\left(x t^{-1 / 2}\right)
$$

and is to satisfy

$$
w_{x x}=w_{t}
$$

in the appropriate region. Such a decomposition is, in some sense, natural in view of the type of singular behavior found in (7d).

Operating with (9) and (10) leads to the ordinary differential equation

$$
\theta^{\prime \prime}(z)+\frac{z}{2} \theta^{\prime}(z)+\theta(z)=0 .
$$


To specify $w(x, t)$, we have at our disposal $\theta(0), \theta^{\prime}(0)$ which we choose with an eye toward making $v(x, t)$ as docile as possible near the origin. Examining the boundary conditions for $v$, we have

$$
\begin{aligned}
v_{x}(0, t) & =\frac{1}{2}-t^{-3 / 2} \theta^{\prime}(0), & & t>0, \\
v(s(t), t) & =t^{-1}\left\{\frac{1}{2}-\theta\left[t^{-1 / 2} s(t)\right]\right\}, & & t>0, \\
v_{x}(s(t), t) & =-t^{-3 / 2} \theta^{\prime}\left[t^{-1 / 2} s(t)\right], & & t>0 .
\end{aligned}
$$

Equation (12) makes it clear that $\theta^{\prime}(0)$ ought to be taken zero. Such a choice having been made, (11) has the power series solution

$$
\theta(z)=\theta(0) \sum_{n=0}^{\infty}(-1)^{n} \frac{n ! z^{2 n}}{(2 n) !} .
$$

Substituting this expression into (13) yields

$$
v(s(t), t)=t^{-1}\left\{\frac{1}{2}-\theta(0)\left[1-\frac{s^{2}(t)}{2 t}+\frac{s^{4}(t)}{12 t^{2}}+\cdots\right]\right\} .
$$

The only chance for good behavior for $v$ as $t \rightarrow 0$ is to choose $\theta(0)=\frac{1}{2}$ in order to kill the singularity caused by $1 / 2 t$, and hope that $s(t)$ turns out to be at least $\theta(t)$ as $t \rightarrow 0$. This seems a reasonable hope in view of the results of Breakwell and Chernoff, whose results will be discussed momentarily.

At this point, we now have that $w$ is the explicit function

$$
w(x, t)=\frac{1}{2 t} \sum_{n=0}^{\infty}(-1)^{n} \frac{n ! x^{2 n}}{(2 n) ! t^{n}}, \quad t, x>0 .
$$

Whence the problem for $B(\xi, \tau), \sigma(\tau)$ has been reduced first to problem (7), and now to this problem for $v(x, t), s(t)$ :

$$
\begin{aligned}
v_{x x} & =v_{t}, & & 0<x<s(t), \\
v_{x}(0, t) & =\frac{1}{2}, & & t>0, \\
v(s(t), t) & =F(s(t), t), & & t>0, \\
v_{x}(s(t), t) & =G(s(t), t), & & t>0,
\end{aligned}
$$

where

$$
\begin{aligned}
F(x, t) & =\frac{1}{2} \sum_{n=1}^{\infty}(-1)^{n+1} \frac{n !}{(2 n) !} x^{2 n} t^{-(n+1)} \\
& =\frac{1}{4} x^{2} t^{-2}-\frac{1}{24} x^{4} t^{-3}+\frac{1}{240} x^{6} t^{-4}+\cdots, \\
G(x, t) & =\frac{1}{2} \sum_{n=1}^{\infty}(-1)^{n+1} \frac{n !}{(2 n-1) !} x^{2 n-1} t^{-(n+1)} \\
& =\frac{1}{2} x t^{-2}-\frac{1}{6} x^{3} t^{-3}+\frac{1}{40} x^{5} t^{-4}+\cdots .
\end{aligned}
$$

This would seem an appropriate juncture to relate problem (18) to the results of Breakwell and Chernoff in [1]. In that paper (in yet other variables from those used 
here) $B$ and $\sigma$ are assumed to have certain series expansions and by use of the boundary conditions, appropriate coefficients are generated to yield an asymptotic solution. When these series are translated into the $x, t$ variables of this paper, one gets the series

$$
\begin{aligned}
u(x, t) & \sim \frac{x}{2}+\frac{1}{2 t} \sum_{n=1}^{\infty}(-1)^{n} \frac{n ! x^{2 n}}{(2 n) ! t^{n}}-\left(\frac{t^{2}}{4}+\frac{t x^{2}}{4}+\frac{x^{4}}{48}\right)+\left(\frac{5 t^{5}}{24}+\cdots\right)+\cdots, \\
s(t) & \sim t^{2}\left(1-\frac{2 t^{3}}{3}+\frac{28 t^{6}}{15}-\frac{184}{21} t^{9}+\cdots\right)
\end{aligned}
$$

There are two important items to observe about these expansions.

(a) The infinite series term in the expansion for $u$ is exactly our function $w$ given in Eq. (17). Except for this sum in (21), the series for $u$ is termwise well-behaved at the origin; this meshes with the hope that $v(x, t)$ itself will behave near $(0,0)$.

(b) The expansion for $s$ given in (21) suggests that $s$ is in fact $\Theta\left(t^{2}\right)$ as $t \rightarrow 0$, which fact makes it reasonable to expect that $F(s(t), t)$ and $G(s(t), t)$ as given in (19) and (20) have finite limits as $t \rightarrow 0$.

The Algorithm. Armed with these suggestions that numerical success is within our grasp, we attack the free boundary problem (18) by applying the method of lines in a manner similar to that given in [4] and [6]. Specifically, (18a) is replaced by the system of ordinary differential equations

$$
w_{n}^{\prime \prime}(x)=\frac{w_{n}(x)-\tilde{w}_{n-1}(x)}{h}, \quad 0 \leqq x \leqq s_{n}, \quad n=1,2, \cdots,
$$

where $s_{n}$ is to be chosen so that

$$
w_{n}^{\prime}(0)=\frac{1}{2}, \quad w_{n}\left(s_{n}\right)=F\left(s_{n}, n h\right), \quad w_{n}^{\prime}\left(s_{n}\right)=G\left(s_{n}, n h\right),
$$

and

$$
\begin{aligned}
\tilde{w}_{n}(x) & =w_{n}(x), & & x \leqq s_{n}, \\
& =F\left(s_{n}, n h\right)+G\left(s_{n}, n h\right)\left(x-s_{n}\right), & & x>s_{n} .
\end{aligned}
$$

(Note. In general, $w_{n}$ will need to be extended beyond $s_{n}$ in order to compute $w_{n+1}$, thus necessitating the extension $\tilde{w}_{n}$ given in (24).)

To initiate this procedure, it is necessary to specify $w_{0}(x)$ on the interval $\left[0, s_{0}\right]$. However, in view of the expectation that $s(0)=0$, it is only necessary to have $\tilde{w}_{0}$ and this is taken to be $\tilde{w}_{0}(x)=x / 2$. This is reasonable in light of condition (18b) and the property that

$$
\lim _{t \rightarrow 0} F(s(t), t)=0
$$

as long as $s(t)=\mathcal{O}(t)$ as $t \rightarrow 0$. In any event, the choice of $\tilde{w}_{0}$ is not critical since its effect on subsequent $w_{n}$ will tend to zero as $h \rightarrow 0$.

Proceeding, Eq. (22) has the explicit solution

$$
w_{n}(x)=A_{n} \varphi_{1}(x)+B_{n} \varphi_{2}(x)-\frac{1}{h} \int_{0}^{x} \tilde{w}_{n-1}(\xi) \varphi_{2}(x-\xi) d \xi
$$

where $\varphi_{1}(x)=\cosh \left(x h^{-1 / 2}\right), \varphi_{2}(x)=\sinh \left(x h^{-1 / 2}\right)$. The three conditions given in (23) 
are to be used to determine $A_{n}, B_{n}, s_{n}$. Using these conditions to eliminate $A_{n}, B_{n}$ from (22), we find that the required $s_{n}$ must be a zero of the function

$$
\Phi_{n}(s)=F(s, n h) \varphi_{2}(s)-h^{-1 / 2} G(s, n h) \varphi_{1}(s)+\frac{1}{2} h^{1 / 2}-h^{-1 / 2} \int_{0}^{s} \tilde{w}_{n-1}(\xi) \varphi_{1}(\xi) d \xi .
$$

An explicit computation algorithm can now be given.

1. The nonlinear function $\Phi_{n}$ in (26) is subjected to one of the extant zero-locating routines; denote the approximated zero by $s_{n}$.

2. With $s_{n}$ in hand, it is a simple matter to compute $A_{n}, B_{n}$ by using (25) and two of the three conditions given in (23).

3. $w_{n}(x)$ can then be computed from (25) for $0 \leqq x \leqq s_{n}$.

Discussion of the Algorithm. To begin with, viewing $\Phi_{n}$ apart from the context of the original problem, there is little (if anything) to suggest that it has any zeroes, much less that it has a unique, positive zero for any $n, h$. Analytically, this is a very difficult problem as can be witnessed in the analyses in [4] and [6] where functions similar but simpler than that in (26) are examined for unique zeroes. Such an analytic investigation will not be made here; nevertheless, from the origins of the problem, we can reasonably expect that $s_{n}$ exists for any $n$ and $h$ and is unique and positive. This conjecture is borne out in the computations.

The method used here to find the approximate endpoints $s_{n}$ is the usual Newton's method, since $\Phi_{n}^{\prime}$ is relatively easy to compute. The subsequent computation of $A_{n}, B_{n}$ and finally of $w_{n}(x)$ is straightforward, with the integrations involved being handled by a Simpson integrator.

Numerical Results. Probably the more useful component of the solution to the original statistical decision problem is the solution boundary, $s(t)$ (née $\sigma(\tau)$ ), rather than the actual values of the Bayes risk itself. After all, it is this boundary which defines the stopping rule-the Bayes risk evaluation is incidental to that rule. Therefore, the bulk of the results cited henceforth will deal with the approximations to $s(t)$.

All the computations were done in single-precision arithmetic on an IBM 360/67. The attendant parameters in these calculations include

(a) Simpson integration step size; $h / 2$ was used in each case,

(b) Maximum error in Newton's method of determining $s_{n}, 10^{-5}$,

(c) Number of terms of $F$ and $G$ used, 12 (cf. (19) and (20)).

(It is reasonably clear that the size of $t$ would influence the number of terms of $F$ and $G$ to be used; the larger $t$, the more terms of $F$ and $G$ to be used; the larger $t$, the more terms of $F$ and $G$ necessary to preserve accuracy. For $t \leqq 2,12$ terms are sufficient and for $t \leqq 1,6$ will actually suffice.)

Table 1 shows the results obtained for $0 \leqq t \leqq .1$, with the concomitant computations from the Breakwell-Chernoff expansion, where the first three terms of that expansion are employed (more terms yields no change). These results for the method of lines are merely corroborative with the asymptotic results.

The more significant contribution from the method of lines is found for larger values of $t$, these results being given in Table 2. It should be noticed that there is (apparent) linear convergence of these boundary estimates. Table 3, on the other 
TABLE 1

\begin{tabular}{ccc}
\hline & $\begin{array}{c}\text { Method of lines boundary } \\
h=.01\end{array}$ & $\begin{array}{c}\text { Breakwell-Chernoff } \\
\text { expansion }\end{array}$ \\
\hline .01 & .0001000 & .0001000 \\
.02 & .0004000 & .0004000 \\
.03 & .0009000 & .0009000 \\
.04 & .0016000 & .0015999 \\
.05 & .0024998 & .0024998 \\
.06 & .0035996 & .0035995 \\
.07 & .0048990 & .0048989 \\
.08 & .0063980 & .0063978 \\
.09 & .0080964 & .0080961 \\
.10 & .0099938 & .0099933 \\
\hline
\end{tabular}

hand, demonstrates the limitations of the Breakwell-Chernoff expansion and at best suggests usable results only for $t$ less than about .5 .

Additional evidence of the divergence of the asymptotic expansion for $s$ is found

TABLe 2

Method of Lines Boundary Estimates

\begin{tabular}{lcccc}
\hline$t$ & $h=.2$ & $h=.1$ & $h=.05$ & $h=.02$ \\
\hline .1 & & .0099970 & .0099957 & .0099943 \\
.2 & .0399482 & .0398675 & .0398273 & .0398054 \\
.3 & & .0888193 & .0886486 & .0885481 \\
.4 & .1558680 & .1548998 & .1544795 & .1542611 \\
.5 & & .2352109 & .2345049 & .2341754 \\
.6 & .3281219 & .3263323 & .3256817 & .3252925 \\
.7 & & .4257654 & .4251320 & .4248957 \\
.8 & .5314652 & .5310651 & .5309301 & .5307290 \\
.9 & & .6406202 & .6410626 & .6411589 \\
1.0 & .7501025 & .7531593 & .7542704 & .7544931 \\
1.1 & & .8682284 & .8699195 & \\
1.2 & .9772512 & .9843462 & .9870185 & \\
1.3 & & 1.101370 & 1.104626 & \\
1.4 & 1.208308 & 1.219378 & 1.223219 & \\
1.5 & & 1.337466 & 1.342154 & \\
1.6 & 1.442169 & 1.455322 & 1.460742 & \\
1.7 & & 1.573937 & 1.574011 & \\
1.8 & 1.675314 & 1.691085 & 1.693958 & \\
1.9 & & 1.807692 & 1.814964 & \\
2.0 & 1.907213 & 1.924348 & 1.941728 & \\
\hline
\end{tabular}


TABLE 3

Breakwell-Chernoff Boundary Estimates

\begin{tabular}{crrrr}
\hline$t$ & 1 term & 2 terms & 4 terms & 6 terms \\
\hline .0 & 0.00 & .0000000 & .0000000 & .0000000 \\
.1 & .01 & .0099933 & .0099934 & .0099934 \\
.2 & .04 & .0397867 & .0397913 & .0397913 \\
.3 & .09 & .0883800 & .0884870 & .0884891 \\
.4 & .16 & .1531733 & .1540291 & .1541040 \\
.5 & .25 & .2291667 & .2321800 & .2322883 \\
.6 & .36 & .3081600 & .3077250 & .2785884 \\
.7 & .49 & .3779534 & .3123113 & -.3085791 \\
.8 & .64 & .4215466 & -.0179218 & -7.225949 \\
.9 & .81 & .4163398 & -1.529703 & -60.27365 \\
1.0 & 1.00 & .3333333 & -6.561902 & -379.3532 \\
\hline
\end{tabular}

in the following family of free boundary problems:

$$
\begin{aligned}
v_{x x}^{n} & =v_{t}^{n}, & & 0<x<s^{n}(t), \\
v_{x}^{n}(0, t) & =\frac{1}{2}, & & t>0, \\
v^{n}\left(s^{n}(t), t\right) & =F\left(S_{n}(t), t\right), & & t>0, \\
v_{x}^{n}\left(s^{n}(t), t\right) & =G\left(S_{n}(t), t\right), & & t>0,
\end{aligned}
$$

where $S_{n}(t)$ is the fixed boundary function comprising the first $n$ terms of the Breakwell-Chernoff expansion; e.g., $S_{1}(t)=t^{2}, S_{2}(t)=t^{2}-\frac{2}{3} t^{5}$, etc. Problems (27) no longer exhibit the unknown boundary $s_{n}(t)$ on the right-hand side of the free boundary conditions; these conditions are now more similar to those found in [6], i.e., of the form

$$
v(s(t), t)=f(t), \quad v_{x}(s(t), t)=g(t) .
$$

Proceeding to solve problem (27) by the same method of lines reveals that for small values of $t$, the solution curves are close to the "assumed" solutions $S_{n}(t)$. However, for $t$ greater than about .6, the process begins to diverge. Of course, if the sequence $S_{n}(t)$ were a convergent one, then the corresponding solutions to problems (27) would converge to that boundary. Since this is not the case, one can only conclude that the Breakwell-Chernoff boundary series is indeed only asymptotic and not convergent for $t$ larger than .6, for example.

Finally, since the function $v(x, t)$ in the solution of (18) has been the victim of some neglect, Table 4 gives approximate values of $v$ for the grid given by $\Delta t=.1$, $\Delta x=.1$ for $t \leqq 1.0$. The values given to the left of the sketched free boundary are at the grid points; the values to the right are the computed values of $v$ at the boundary, the boundary points being given in Table 2 under the column $h=.05$. Table 4 was computed with $h=.05$ and integration step size of .025 . To give some notion of the 


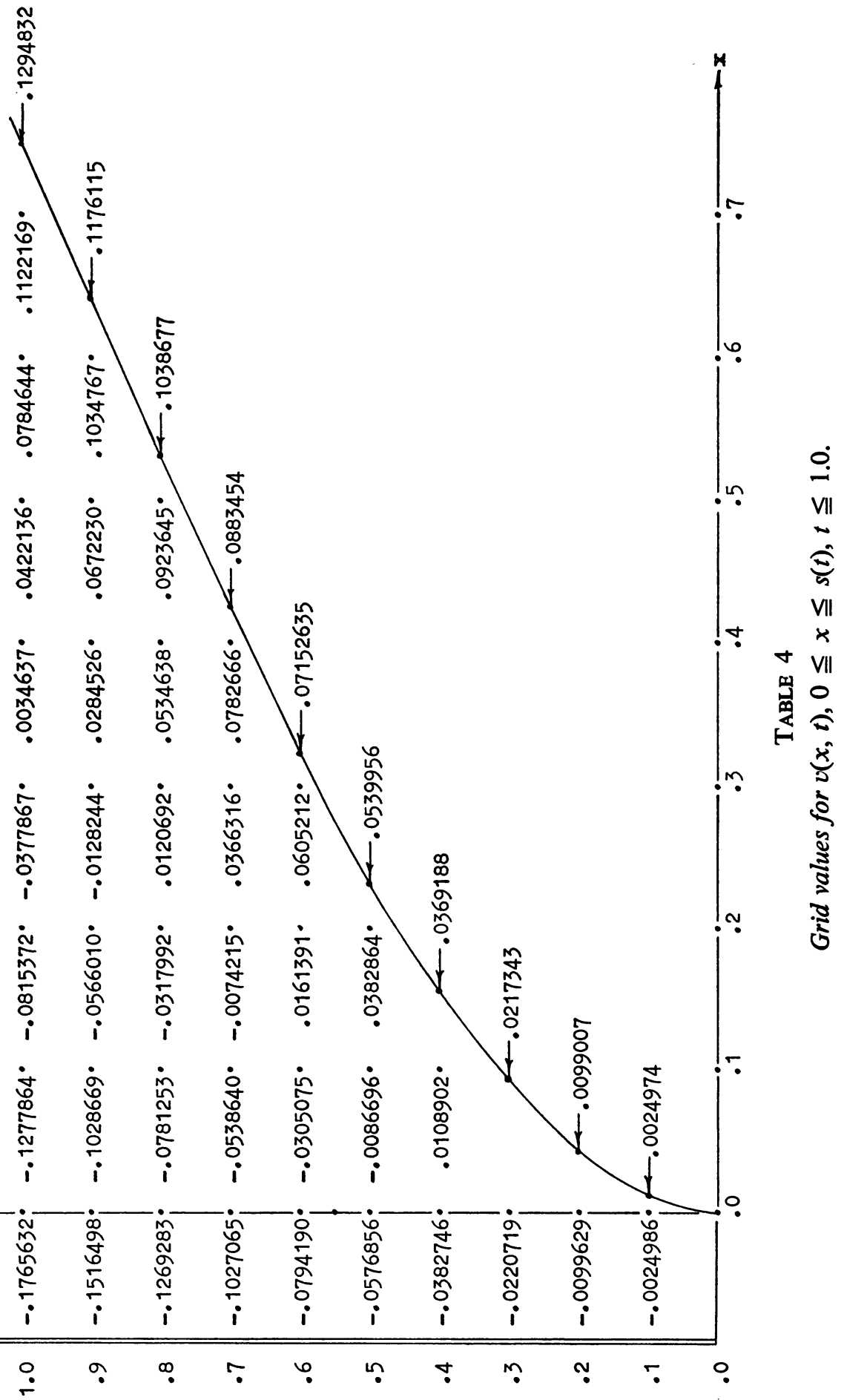


speed of the method, the results given in Table 4 were tabulated in less than 30 seconds on the IBM $360 / 67$.

Department of Mathematics and Statistics

The University of New Mexico

Albuquerque, New Mexico 87106

1. John Breakwell \& Herman Chernoff, "Sequential tests for the mean of a normal distribution. II. (large t)," Ann. Math. Statist., v. 35, 1964, pp. 162-173. MR 28 \#1679.

2. Herman Chernoff, Sequential Tests for the Mean of a Normal Distribution, Fourth Berkeley Sympos. on Math. Statist. and Prob., vol. 1, Univ. of California Press, Berkeley, Calif., 1961, pp. 79-91. MR 24 \#A1788.

3. HERMAN CHERNOFF, "Sequential tests for the mean of a normal distribution. III. (small t)," Ann. Math. Statist., v. 36, 1965, pp. 28-54. MR 30 \#680.

4. NGUYEN DINH CHI (THI), "On a free boundary problem for a parabolic equation," Vestnik Moskov. Univ. Ser. Math. Mech., No. 2, 1966, pp. 40-54. (Russian)

5. ERICH ROTHE, "Two dimensional parabolic boundary value problems as the limit of one dimensional boundary value problems," Math. Ann., v. 102, 1930, pp. 650-670. (German)

6. G. G. SACKETT, "An implicit free boundary problem for the heat equation," SIAM J. Numer. Anal., v. 8, 1971.

7. B. Sherman, Some Comments on Free Boundary Problems for Parabolic Equations Arising in Statistical Decision Theory, Rocketdyne Research Report \#66-24, 1966. 